\title{
ON RANDOMIZED REINSURANCE CONTRACTS
}

\author{
HANSJÖRG ALBRECHER AND ARIAN CANI
}

\begin{abstract}
In this paper we discuss the potential of randomizing reinsurance treaties for efficient risk management. While it may be considered counter-intuitive to introduce additional external randomness in the determination of the retention function for a given occurred loss, we indicate why and to what extent randomizing a treaty can be interesting for the insurer. We illustrate the approach with a detailed analysis of the effects of randomizing a stop-loss treaty on the expected profit after reinsurance in the framework of a one-year reinsurance model under regulatory solvency constraints and cost of capital considerations.
\end{abstract}

\section{Introduction And Motivation}

Reinsurance is a classical tool for the risk management of an insurance company. Among the many motivations for entering a reinsurance treaty, one that is of particular importance from an actuarial point of view is its function as a risk transfer, as it helps to reduce the risk exposure of the insurer and hence to stabilize the business (see e.g. Albrecher et al. [1] for a recent overview). Passing on some part of the insurance risk to a reinsurance company comes at the expense of paying a respective reinsurance premium, which reduces the potential profits, so that there is a tradeoff as to how much reinsurance is desirable for the insurance company. The solution naturally depends on the criteria that are used to quantify the performance of the retained portfolio as well as the pricing rule that is applied by the reinsurer for accepting the ceded part of the risk. Historically, the study of optimal reinsurance treaties can be traced back to the seminal papers of Borch [6] and Arrow [2] and has been an active research field both for academics and practicioners since then. Borch [6] showed that a stop-loss treaty minimizes the variance of the insurer's retained loss when the reinsurance premium is prespecified and determined according to an expected value premium principle. In the framework of risk-averse utility functions, Arrow [2] established that such a stop-loss contract more generally maximizes the expected utility of the terminal wealth of the insurer. Over the following decades, there were many contributions in the field, generalizing these classical results for more intricate optimality criteria and/or more general premium principles (see for

Financial support by the Swiss National Science Foundation Project 200021_168993/1 is gratefully acknowledged. 
instance Gajek \& Zagrodny [18], Kaluszka [22], Centeno \& Guerra [20] as well as Tan et al. [26], Malamud et al. [25] and Chi et al. [13] for some recent contributions, and $[1$, Ch.8] for a survey).

Prompted by the recent insurance regulatory developments aiming at the harmonization of risk assessment procedures, the Value-at-Risk (VaR) and Conditional-TailExpectation (CTE) became benchmark risk measures to reflect risk and subsequently determine capital requirements of an insurance company. Consequently, considerable attention has turned to embedding these two risk measures in the study of optimal reinsurance models. Cai \& Tan [8] derive analytically the optimal retention of a stoploss reinsurance treaty which minimizes the VaR and CTE of the insurer's remaining risk exposure under the expected value premium principle. These results were later generalized by Cai et al. [9] who examine optimal reinsurance schemes within the class of increasing convex functions. Using a geometric approach, Cheung [10] simplifies the arguments in Cai et al. [9] and identifies the stop-loss treaty as optimal also when the expected value premium principle is replaced by Wang's premium principle in the VaR-minimization problem. Within the setting of minimizing the VaR and CTE of the total retained loss of the insurer, Chi \& Tan [14] determine the optimal reinsurance contract among a larger class of admissible reinsurance schemes, see also Chi [11] and Chi \& Tan [15] for further extensions.

All the reinsurance forms considered above are of a deterministic form, i.e. for a risk $X$ there is a fixed pre-defined function $r(X)$ that determines how much of the risk $X$ is retained by the first-line insurer. While this is a traditional and intuitive way to specify the risk participation of the reinsurer, the question arises whether there could not exist situations in which additional randomness in the specification of $r(\cdot)$ could be advantageous. For instance, consider a reinsurance treaty that provides stop-loss coverage of the following form: at the end of the year a coin is flipped, and if the outcome is "Heads", then the reinsurer participates in the claim payment according to a stop-loss treaty with some pre-defined retention $d$, otherwise no reinsurance is provided. An immediate generalization of such a mechanism is to draw the realized retention level independently from a more general distribution (it will, however, turn out that a two-point distribution can not be outperformed for the optimization criteria considered below).

Guerra \& Centeno [21] in fact used randomized treaties as a mathematical tool to identify optimal reinsurance forms under a general class of risk measures and premium principles, when the criterion is to minimize the risk measure of the retained risk exposure. As in other mathematical contexts (like the identification of Nash equilibria in game theory), this (in a certain sense) implicit 'convexification' allows 
to show the existence of an optimal strategy among such an enlarged set of admissible reinsurance forms. One can then (for the same premium) achieve an identical resulting cumulative distribution function of the retained loss through a deterministic treaty, which finally is the optimal reinsurance form (see [21] for details). While the latter argument at first glance seems to render the practical implementation of randomized treaties unnecessary, the 'equivalent' deterministic treaty can have unfavourable properties (like non-monotonicities or even discontinuities). Also, as will be discussed later, randomization of treaties may be simpler and may have some particular advantages to avoid moral hazard problems. We therefore in this paper would like to take up the discussion of randomized reinsurance treaties from a more practical perspective, namely to study how randomization of classical treaties possibly increases the efficiency of risk sharing, and how it affects the resulting loss distribution. Eventually, randomization can be seen as an alternative method to reshape the loss distribution of the insurer.

We would like to point out that Gajek and Zagrodny [19] also discovered randomized reinsurance treaties as 'curious' possible solutions in the presence of discrete loss variables when the goal is to minimize the ruin probability of an insurer and there is a constraint on the available reinsurance premium, a problem which they nicely linked to the Neyman-Pearson lemma in statistical hypothesis testing (and in that case the performance of these randomized treaties can not be matched by a deterministic treaty). This connection between optimal reinsurance and the design of most powerful tests in statistics was recently studied in more detail in Lo [24].

In order to maintain transparency of the ideas involved, we prefer in this paper to restrict our analysis to a simple stop-loss treaty on the aggregate loss of an insurance portfolio, and randomize it according to an independent mechanism (a lottery) that after the aggregate claim has been settled - determines the retention of the stop-loss cover. Should such a randomized reinsurance cover be realized in practice, one could for instance think of a random experiment that both parties agree upon, possibly in the presence of a notary. At a first glance, such a random mechanism to determine the final participation of the reinsurer may seem unnatural, not the least because a reinsurer intends to help the insurer in adverse cases of large claims. However, reinsurance as well as direct insurance in the first place, is about efficiently dealing with risks, and if a non-standard reinsurance form is useful to reshape the loss distribution of the insurer in a cost-efficient and simple way, it may be worthwhile to be considered. From an insurer's viewpoint, such an uncertainty in the reinsurance cover could be compared with hearing about an event (like a natural catastrophe), but not yet knowing what the implications for the actual claim payments to policyholders will be, or also with the uncertainty until the full development of some 
claim. In the randomization case one knows the original claim size but does not yet know how much of it will finally remain with the insurer, so the main difference being that in the latter case the randomization is introduced artificially (but for efficiency reasons). Such additional introduced randomness can in fact be observed in some reinsurance treaties already implemented in practice, where the coverage is made dependent on a financial index or the financial performance of the insurance company itself (like in certain finite-risk reinsurance setups, see e.g. Culp [16]). For the 'marginal' analysis of the insurance liabilities, this introduced randomness can be interpreted as independent of the insurance risks.

The criterion for studying the effectiveness of reinsurance contracts in this paper will be the one of maximizing expected profit after reinsurance, taking into account capital costs from the resulting solvency constraint for some fixed cost-of-capital rate, which goes back to Kull [23]. For a comparison to other criteria recently popular in the literature on optimal reinsurance forms, we refer to Remark 2.1 or [1, Ch.8]. For the sake of simplicity, we focus here solely on the insurance risk (no market risk, counterparty risk etc.) in a one-period framework and assume that there is no settlement delay of claims. Note, however, that a random reinsurance treaty that with a certain probability provides no cover can also be interpreted in terms of default risk, cf. Section 2. As a risk measure for the determination of the required solvency capital, we restrict the analysis to the VaR. Preferences induced by cost-of-capital considerations and VaR in reinsurance are e.g. studied by Boonen et al. [5], who use a bilateral bargaining approach to identify the optimal risk sharing in the context of optimal reinsurance contract design. In [12], Chi investigates the problem of finding a reinsurance form that minimizes the risk-adjusted value of an insurer's liability, where the valuation is performed using a cost-of-capital approach.

As amply emphasized in the literature, the choice of $\mathrm{VaR}$ in practice is questionable for several reasons, in the present context notably because it encourages excessive protection of medium-sized claims rather than large ones (see also Basak \& Shapiro [3], Bernard \& Tian [4] and Guerra \& Centeno [21]). Yet this risk measure is currently implemented by many regulators and it seems that this will continue to be the case in the near future. The results below may also reinforce from a methodological point of view the doubtfulness of the use of VaR in practice for measuring risk in this context.

The rest of the paper is organized as follows. In Section 2, we introduce the particular randomized stop-loss reinsurance treaty, the model and the objective function. Section 3 derives the optimal randomized treaty under an expected value principle for the reinsurance premium and discusses some concrete cases in more detail. In Section 4, it is then studied which retention level of a stop-loss contract is optimal for any given probability level of the randomization procedure, which gives some 
additional insight in the structure of the problem. Section 5 gives some numerical illustrations of the potential of randomizing classical contracts. Moreover, in Section 6 we compare the randomized stop-loss treaties with (deterministic) bounded stoploss treaties, as the two share certain similarities. Finally, Section 7 contains some further practical considerations and conclusions.

\section{THE MODEL}

In this paper we will study the effects of randomizing a simple stop-loss treaty. Let the random variable $X$ denote the agregate loss that the insurer faces over one year. For convenience, let us assume here that $X$ is continuous (but note that the more general case can be handled analogously). Let further $Y$ be a Bernoulli random variable, independent of $X$, with $\mathbb{P}(Y=1)=p$ and $\mathbb{P}(Y=0)=1-p$ for some fixed $p(0 \leq p \leq 1)$. Consider now a randomized reinsurance contract of the form

$$
r(X)=r(X, Y, d)= \begin{cases}\min (X, d), & \text { if } Y=1 \\ X, & \text { if } Y=0\end{cases}
$$

where $r(X)$ denotes the retained loss of the insurer after reinsurance. That is, after the realization of $X$ there is a random experiment (which is independent of the outcome of $X$ ) that decides whether the reinsurance coverage of $X$ is according to a $\mathrm{SL}$ treaty with retention $d$ or whether no reinsurance takes place. As the dependence on $Y$ should be clear from the above construction, for the sake of notational simplicity we will write $r(X)$ or $r(X, d)$ in the sequel, and not write the dependence on $Y$ explicitly throughout.

Here, the value of $p$ should be viewed as a controllable parameter, i.e. its value can be modified by adjusting the underlying independent random experiment. Note that an alternative interpretation of the random retention (1) is in terms of default risk, i.e. the situation where the reinsurer defaults with probability $1-p$ and can not provide the contracted loss participation (see e.g. Cai et al. [7] for a study of optimal reinsurance arrangements in such a context). In that case the parameter $p$ would not be controllable, but possibly be accessible by a rating mechanism. The results in this paper for the treaty above can indeed be interpreted as a contribution to that stream of literature, however both the motivation and the focus of the present approach are different, and we will not pursue here the connection to default risk models further.

While we later will allow for a random retention following a more general distribution than only the two-point distribution on $\{d, \infty\}$, the latter in fact will turn out to be optimal, so we focus the analysis first on this case. The resulting cumulative 
distribution function (c.d.f.) for the insurer then is

$$
F_{r(X)}(x)= \begin{cases}F_{X}(x), & 0 \leq x<d, \\ p+(1-p) F_{X}(x), & x \geq d,\end{cases}
$$

cf. Figure 1. For the survival function $\bar{F}_{r(X)}(x)=1-F_{r(X)}(x)$, we equivalently have

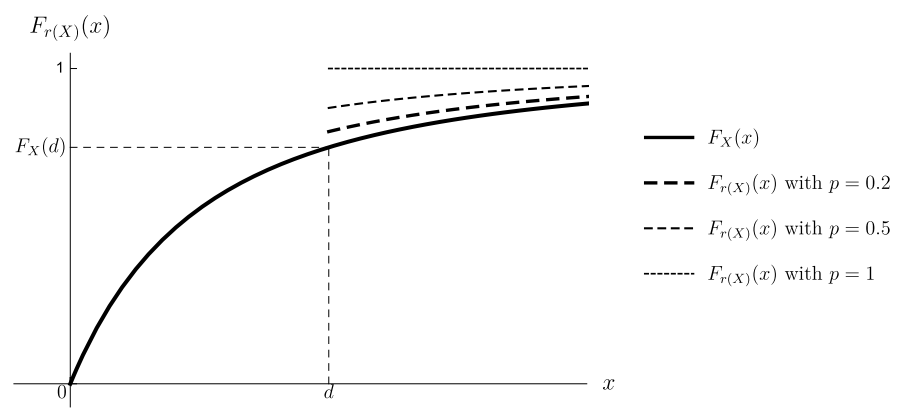

Figure 1. $F_{r(X)}$ for various values of $p$ (for $x<d$ all c.d.f.'s coincide)

$$
\bar{F}_{r(X)}(x)= \begin{cases}\bar{F}_{X}(x), & 0 \leq x<d, \\ (1-p) \bar{F}_{X}(x), & x \geq d .\end{cases}
$$

From the latter expression, one easily deduces the expected retained claim amount

$$
\mathbb{E}[r(X, d)]=\mathbb{E}[X]-p \int_{d}^{\infty} \bar{F}_{X}(x) d x .
$$

Let $\pi(X)$ denote the total premium that the first-line insurer received from policyholders for accepting the aggregate risk $X$. Following a suggestion of Kull [23], one can consider the annual loss

$$
\text { Loss }=X-\pi(X)+r_{C o C} \cdot \rho(\text { Loss }),
$$

where $r_{C o C} \cdot \rho($ Loss $)$ reflects capital costs, with $r_{C o C}$ denoting a cost-of-capital rate and $\rho$ a solvency risk measure. For a positively homogeneous and translation-invariant risk measure $\rho$, this leads to

$$
\rho(\operatorname{Loss})=\frac{\rho(X)-\pi(X)}{1-r_{C o C}}
$$

and consequently the annual profit (i.e. negative loss) is given by

$$
\frac{\pi(X)}{1-r_{C o C}}-X-\frac{r_{C o C}}{1-r_{C o C}} \cdot \rho(X)
$$

(note that this approach for incorporating solvency capital requirements focuses on the current-year insurance risk only, which could then be complemented by market 
risk, counterparty risk, multi-year loss development patterns etc., see [1, Ch.8] for further details). If a reinsurance treaty of the form (1) is entered for a premium $\pi_{R}(d)$, then (3) changes into

$$
Z(d)=\frac{\pi(X)-\pi_{R}(d)}{1-r_{C o C}}-r(X, d)-\frac{r_{C o C}}{1-r_{C o C}} \cdot \rho(r(X, d)) .
$$

In (4), the dependence on $p$ is implicit, and the notations $Z(d)$ and $Z(d, p)$ will be used interchangeably in the sequel. As a performance measure of a reinsurance treaty, we will in this paper choose the resulting expected annual profit $\mathbb{E}(Z(d))$, since it combines the solvency aspect with the profitability considerations in an intuitive way. Furthermore, to simplify calculations we will assume an expected value principle for the reinsurance premium (with relative safety loading $\theta>0$ ):

$$
\pi_{R}(d)=(1+\theta) \mathbb{E}[X-r(X, d)]=(1+\theta) p \int_{d}^{\infty} \bar{F}_{X}(x) d x .
$$

For the risk measure $\rho$, we choose the Value-at-Risk (VaR) at level $1-\alpha$ and use the notation

$$
\rho(X)=\operatorname{VaR}_{\alpha}(X)=\inf \left\{x: \bar{F}_{X}(x) \leq \alpha\right\}, \quad \alpha \in(0,1) .
$$

Since the constant $\alpha$ reflects the percentage of risk tolerance, it will typically be small.

This leads to the optimization problem

$$
\max _{0 \leq p \leq 1, d \geq 0} \mathbb{E}[Z(d, p)]
$$

with

(6)

$\mathbb{E}[Z(d, p)]=\frac{\pi(X)}{1-r_{C o C}}-\mathbb{E}[X]-\frac{r_{C o C}}{1-r_{C o C}}\left(\left(1+\frac{\theta}{r_{C o C}}\right) p \int_{d}^{\infty} \bar{F}_{X}(x) d x+\operatorname{VaR}_{\alpha}(r(X, d))\right)$.

In view of (6), the optimization problem (5) can be reformulated as

$$
g\left(d^{*}, p^{*}\right):=\min _{d \geq 0,0 \leq p \leq 1} g(d, p)
$$

where

$$
g(d, p):=\left(1+\frac{\theta}{r_{C o C}}\right) p \int_{d}^{\infty} \bar{F}_{X}(x) d x+\operatorname{VaR}_{\alpha}(r(X, d)) .
$$

Clearly, the trade-off to consider is to reduce the capital costs with a not too expensive reinsurance premium, and we will see in the sequel that under the present assumptions this trade-off can be more efficiently resolved introducing randomized reinsurance forms, i.e. $0<p<1$. 
Remark 2.1. For general reinsurance treaties $r(X)$, a general premium principle $\pi_{R}$ and risk measure $\rho$ the above optimization criterion leads to minimizing

$$
\pi_{R}(X-r(X))-\left(1-r_{C o C}\right) \mathbb{E}[X-r(X)]+r_{C o C} \cdot \rho(r(X))
$$

over all admissible $r(X)$. Note that this in general differs from the purely risk-averse objective function $\rho\left(\pi_{R}(X-r(X))+r(X)\right)$ used by Cai \& Tan [8] and several subsequent papers in the literature, but in case of the expected value premium principle and translation invariance of $\rho$ the two can be identified for a modified value of the safety loading coefficient (and hence different weighting), cf. [1, Sec.8.4] for details.

\section{The OPTIMIZATION PROBLEM}

In view of (8), it is clear that in our setting only retention values $d<\bar{F}_{X}^{-1}(\alpha)$ are of interest, as otherwise the reinsurance treaty does not improve $\operatorname{VaR}_{\alpha}(X)$ and therefore it is better not to take reinsurance at all (and keep the saved reinsurance premium for profit). For each potentially optimal candidate $d$, let

$$
p(d):=1-\frac{\alpha}{\bar{F}_{X}(d)},
$$

be the value of $p$ such that one has $F_{r(X, d)}(d)=p+(1-p) F_{X}(d)=1-\alpha$. Then, for each candidate $d$, the optimal $p$ must satisfy $p \leq p(d)$, otherwise the solvency capital requirement is over-fulfilled in the sense that the same level of $\operatorname{VaR}_{\alpha}(r(X, d))$ could be achieved for a lower reinsurance premium simply by decreasing $p$ to (9) (cf. Figure 2).

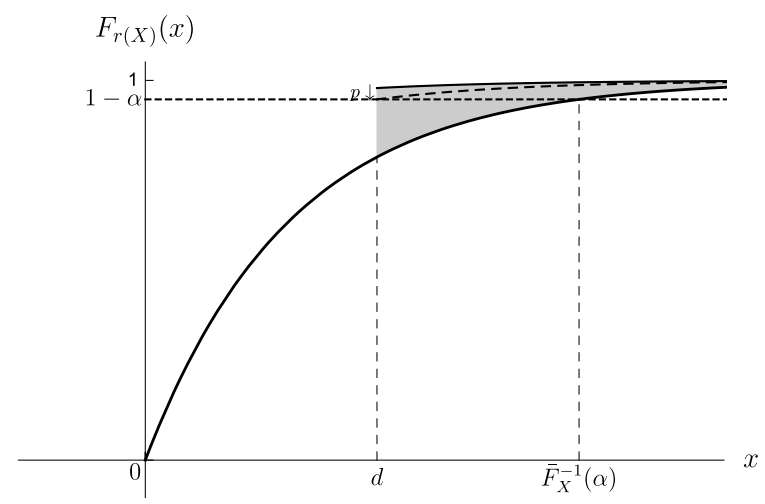

Figure 2. Transition from an inefficient to a possibly efficient solution in a randomized stop-loss treaty 
Assume now that for the candidate $d, \tilde{p}<p(d)$ is optimal, in which case $\operatorname{VaR}_{\alpha}(r(X, d))=$ $\tilde{d}>d$. One could attain the same level of $\operatorname{VaR}_{\alpha}(r(X, d))$ by increasing $d$ (i.e. transferring the location of the jump of $\left.F_{r(X, d)}\right)$ to the level $\widetilde{d}>d$ such that $p+(1-p) F_{X}(\widetilde{d})=$ $1-\alpha$ holds, so that the same value $\operatorname{VaR}_{\alpha}(r(X, d))=\operatorname{VaR}_{\alpha}(r(X, \widetilde{d}))=\bar{F}_{X}^{-1}\left(\frac{\alpha}{1-p}\right)$ is achieved by a smaller reinsurance premium.

Consequently, the original $d$ could not have been optimal for the overall optimization problem. One can hence fix $p(d)$ according to (9) and the optimization problem (7) reduces to the one-dimensional problem

$$
\min _{0 \leq d \leq \bar{F}_{X}^{-1}(\alpha)} g\left(d, 1-\alpha / \bar{F}_{X}(d)\right) .
$$

Note that if the optimal retention is the right-end point of this interval, i.e. $d^{*}=$ $\bar{F}_{X}^{-1}(\alpha)$, the corresponding probability is $p^{*}=p\left(d^{*}\right)=0$, which means no reinsurance (this also corresponds to $d=\infty$ for any $p$ ) and the resulting objective function then is $\operatorname{VaR}_{\alpha}(X)=\bar{F}_{X}^{-1}(\alpha)$.

It is clear from $(9)$, but useful to note for later purposes, that we always have $p(d) \leq$ $1-\alpha$, with equality for $d=0$.

Problem (10) translates into

$$
\min _{0 \leq d \leq \bar{F}_{X}^{-1}(\alpha)}\left(1+\theta / r_{C o C}\right)\left(1-\alpha / \bar{F}_{X}(d)\right) \int_{d}^{\infty} \bar{F}_{X}(x) d x+d .
$$

This can also be expressed in terms of the mean-excess function $e_{X}(u)=\mathbb{E}(X-u \mid X>$ $u$ ) and the pure reinsurance premium $\pi_{S L}(d)=\int_{d}^{\infty} \bar{F}_{X}(x) d x$ of a classical unbounded stop-loss contract (i.e. $p=1$ ):

$$
\min _{0 \leq d \leq \bar{F}_{X}^{-1}(\alpha)}\left(1+\theta / r_{C o C}\right)\left(\pi_{S L}(d)-\alpha \cdot e_{X}(d)\right)+d .
$$

One observes that the shape of this function strongly depends on the distribution of the loss variable $X$ and a general analysis is difficult. In any case, a particular candidate for an optimal retention $d$ is the solution of the equation

$$
\left(1+\theta / r_{C o C}\right)\left(\bar{F}_{X}(d)+\alpha \cdot e_{X}^{\prime}(d)\right)=1 .
$$

Example 3.1. If $X$ is exponentially distributed with parameter $\nu$, then $e_{X}(d)=1 / \nu$ and the solution of (12) is indeed

$$
d=\frac{1}{\nu} \log \left(1+\theta / r_{C o C}\right) .
$$

Since in this case $\bar{F}_{X}^{-1}(\alpha)=\frac{1}{\nu} \log (1 / \alpha)$, the solution of the overall optimization problem (5) is the following: If $\frac{1}{\alpha}>1+\theta / r_{C o C}$, then the optimal retention $d^{*}$ is given by (13) together with the corresponding $p^{*}=1-\alpha\left(1+\theta / r_{C o C}\right)(c f .(9))$. If 
$\frac{1}{\alpha} \leq 1+\theta / r_{C o C}$, then $d^{*}=\infty$, i.e. no reinsurance of the form (1) should be taken (in this case the reinsurance premium, through the loading $\theta$, is too expensive or the cost-of-capital rate is too small relative to the solvency quantile $\alpha$, so that reinsurance is not efficient).

Example 3.2. If $X$ follows a shifted Pareto distribution, i.e.

$$
F_{X}(x)=1-\left(\frac{\xi}{x+\xi}\right)^{1 / \gamma}, \quad \xi>0 ; \gamma<1,
$$

then $e_{X}(d)=\frac{d+\xi}{\frac{1}{\gamma}-1}$, and the solution of (12) is given by

$$
d=\xi\left(\left(\frac{1}{1+\frac{\theta}{r_{C o C}}}-\frac{\alpha}{\frac{1}{\gamma}-1}\right)^{-\gamma}-1\right) .
$$

Since $\bar{F}_{X}^{-1}(\alpha)=\xi\left(\alpha^{-\gamma}-1\right)$, the solution of the overall optimization problem (5) is as follows: If $\frac{1}{\alpha}(1-\gamma)>1+\theta / r_{C o C}$, then $d^{*}$ is given by (14) together with

$$
p^{*}=\frac{\frac{1}{\gamma}\left(1-\alpha\left(1+\frac{\theta}{r_{C o C}}\right)\right)-1}{\frac{1}{\gamma}-\alpha\left(1+\frac{\theta}{r_{C o C}}\right)-1} .
$$

If $\frac{1}{\alpha}(1-\gamma) \leq 1+\theta / r_{C o C}$, then it is optimal to take no reinsurance.

Example 3.3. If $X$ is uniformly distributed in $[0, b]$, one has $e_{X}(d)=1 / 2(b-d)$ for $d<b$, and

$$
d=b\left(1-\left(\frac{1}{1+\frac{\theta}{r_{C o C}}}+\frac{\alpha}{2}\right)\right)
$$

solves (12). Because $\bar{F}_{X}^{-1}(\alpha)=b(1-\alpha)$, the solution of the overall optimization problem (5) then reads the following: If $\frac{2}{\alpha}>1+\theta / r_{C o C}$, then $d^{*}$ is given by (15) together with

$$
p^{*}=\frac{\frac{1}{1+\frac{\theta}{r_{C o C}}}-\frac{\alpha}{2}}{\frac{1}{1+\frac{\theta}{r_{C o C}}}+\frac{\alpha}{2}} .
$$

If $\frac{2}{\alpha} \leq 1+\theta / r_{C o C}$, then $d^{*}=\infty$, i.e. the expected profit is maximized when no reinsurance is purchased.

Remark 3.1. One could equivalently have started the analysis from the viewpoint of choosing candidate values $p$ first. In much the same way as above, let, for a given 
candidate $p \leq 1-\alpha$,

$$
d(p)=\bar{F}_{X}^{-1}\left(\frac{\alpha}{1-p}\right)
$$

be the choice of $d$ so that $F_{r(X, d)}(d)=p+(1-p) F_{X}(d)=1-\alpha$. Here, it is clear that only $p \leq 1-\alpha$ can be optimal, since for any candidate $d$, the resulting $\operatorname{VaR}_{\alpha}(r(X, d))$ would not be improved (in comparison to the choice of $p=1-\alpha$ ) by choosing $p>1-\alpha$, which is more expensive (note that in particular a classical unbounded stop-loss contract $(p=1)$ can not be optimal for $(5)$, since reinsurance beyond the solvency quantile is not efficient). Clearly, for each candidate $p, d<d(p)$ cannot be optimal since for such values of $d, \operatorname{VaR}_{\alpha}(r(X, d))$ remains constant, hence a larger $d$ is preferable. Conversely, if $\tilde{d}>d(p)$ is optimal, then $\operatorname{VaR}_{\alpha}(r(X, \tilde{d}))=$ $\tilde{d}>d(p)$. However, the same Value-at-Risk $\tilde{d}$ could be attained by choosing $p$ such that $F_{r(X, \tilde{d})}(\tilde{d})=p+(1-p) F_{X}(\tilde{d})=1-\alpha$, hence the original $p$ could not have been optimal at the first place. Altogether, fixing (16), the optimization problem (7) then reduces to the one-dimensional problem

$$
\min _{0 \leq p \leq 1-\alpha}\left(1+\frac{\theta}{r_{C o C}}\right) p \int_{\bar{F}_{X}^{-1}\left(\frac{\alpha}{1-p}\right)}^{\infty} \bar{F}_{X}(x) d x+\bar{F}_{X}^{-1}\left(\frac{\alpha}{1-p}\right),
$$

which has a less intuitive form than (11).

In fact, the randomized treaty studied in this section (based on a two-point distribution on $\{d, \infty\})$ is the optimal treaty among all randomized stop-loss treaties with arbitrary distribution for the random retention:

Theorem 3.1. Let $\mathfrak{R}$ be the set of all stop-loss treaties with a random retention level $D$ with c.d.f. $F_{D}$, where $D$ is independent of $X$. Assume that the reinsurance premium is determined by $\pi_{R}(\mathcal{R})=(1+\theta) \mathbb{E}(\mathcal{R})$ for every $\mathcal{R} \in \mathfrak{R}$. Then the expected value of

$$
\mathbb{E}\left(\frac{\pi(X)-\pi_{R}(X-\mathcal{R})}{1-r_{C o C}}-(X-\mathcal{R})-\frac{r_{C o C}}{1-r_{C o C}} \cdot \operatorname{Va} R_{\alpha}(X-\mathcal{R})\right)
$$

is maximized for

$$
D= \begin{cases}d^{*}, & \text { with prob. } p^{*}, \\ \infty, & \text { with prob. } 1-p^{*} .\end{cases}
$$

Proof. Consider the optimal two-point solution (18). Since the reinsurance premium follows an expected value principle, it is proportional to the grey area in Figure 2. Whenever another random variable $D$ leads to a different value of $\operatorname{VaR}_{\alpha}(X-\mathcal{R})$, the two-point distribution on $\left\{\operatorname{VaR}_{\alpha}(X-\mathcal{R}), \infty\right\}$ with the respective value $p\left(\operatorname{VaR}_{\alpha}(X-\right.$ $\mathcal{R})$ ) according to $(9)$ can generate the same VaR value, but for a cheaper reinsurance 
premium. Hence the optimal choice of $d^{*}$ (together with $p^{*}$ ) can not be outperformed by any other random variable $D$ that is independent of $X$.

\section{Optimizing the REtention fOR FiXed $p$}

While the determination of the optimal pair $\left(d^{*}, p^{*}\right)$ is already studied in Section 3, we now identify the optimal retention level for an arbitrary (possibly non-optimal) given probability level $p$. This will give some additional insight into the nature and consequences of the randomization procedure. We will now also allow $F_{X}(0)>0$ (which we refrained from in the previous sections for the sake of clarity of exposition, but which may for instance be relevant in some catastrophe insurance portfolios). Let us start with some general observations.

4.1. Preliminary properties. First, observe that if $1-\alpha \leq F_{X}(0)$, then clearly $\operatorname{VaR}_{\alpha}(r(X, d))=0$ for all $d \geq 0$ in which case the expected profit in (5) is trivially maximized for $p=0$, i.e. no reinsurance. We hence assume $\alpha<\bar{F}_{X}(0)$ in the following.

If $1-\alpha<F_{X}(d)$, i.e. $d>\bar{F}_{X}^{-1}(\alpha)$, then $\operatorname{VaR}_{\alpha}(r(X, d))=\operatorname{VaR}_{\alpha}(X)=\bar{F}_{X}^{-1}(\alpha)$. In this case the retention $d$ exceeds the $\mathrm{VaR}$ of the original (and also the retained) risk, so reinsurance is again not of interest, as the reinsurance premium reduces the expected profit, but does not lower the capital costs.

Next, for $F_{X}(d) \leq 1-\alpha \leq p+(1-p) F_{X}(d)$, i.e. $(1-p) \bar{F}_{X}(d) \leq \alpha \leq \bar{F}_{X}(d)$, we have $\operatorname{VaR}_{\alpha}(r(X, d))=d$.

Finally, in case $1-\alpha>p+(1-p) F_{X}(d)$, i.e. $d<\bar{F}_{X}^{-1}\left(\frac{\alpha}{1-p}\right)$, we have $\operatorname{VaR}_{\alpha}(r(X, d))=$ $\bar{F}_{X}^{-1}\left(\frac{\alpha}{1-p}\right)$.

Summarizing this differently, for each fixed retention $d \geq 0$, the Value-at-Risk of the retained loss amount reads

$$
\operatorname{VaR}_{\alpha}(r(X, d))=\bar{F}_{X}^{-1}(\alpha)
$$

for $p=0$,

$$
\operatorname{VaR}_{\alpha}(r(X, d))= \begin{cases}\bar{F}_{X}^{-1}\left(\frac{\alpha}{1-p}\right), & 0 \leq d<\bar{F}_{X}^{-1}\left(\frac{\alpha}{1-p}\right) \\ d, & \bar{F}_{X}^{-1}\left(\frac{\alpha}{1-p}\right) \leq d \leq \bar{F}_{X}^{-1}(\alpha), \\ \bar{F}_{X}^{-1}(\alpha), & d>\bar{F}_{X}^{-1}(\alpha)\end{cases}
$$


for $p \in\left(0,1-\frac{\alpha}{\bar{F}_{X}(0)}\right)$, and

$$
\operatorname{VaR}_{\alpha}(r(X, d))= \begin{cases}d, & 0 \leq d \leq \bar{F}_{X}^{-1}(\alpha) \\ \bar{F}_{X}^{-1}(\alpha), & d>\bar{F}_{X}^{-1}(\alpha)\end{cases}
$$

for $p \in\left[1-\frac{\alpha}{\bar{F}_{X}(0)}, 1\right]$. Note that in the latter case the Value-at-Risk is bounded by $d$ for any $d \geq 0$ (cf. Figure 3 (right)), whereas it exceeds $d$ in the range $0 \leq d<$ $\bar{F}_{X}^{-1}\left(\frac{\alpha}{1-p}\right)$ in the case $p<1-\frac{\alpha}{\bar{F}_{X}(0)}$ (cf. Figure 3 (left)). One observes that the domain of the Value-at-Risk as a function of $d$ is enlarged for increasing $p$, reaching the situation on the right-hand picture when $p$ tends to $1-\alpha / \bar{F}_{X}(0)$ (and, conversely, for $p \rightarrow 0$ the constant $\bar{F}_{X}^{-1}(\alpha)$ is reached for all values of $\left.d\right)$.
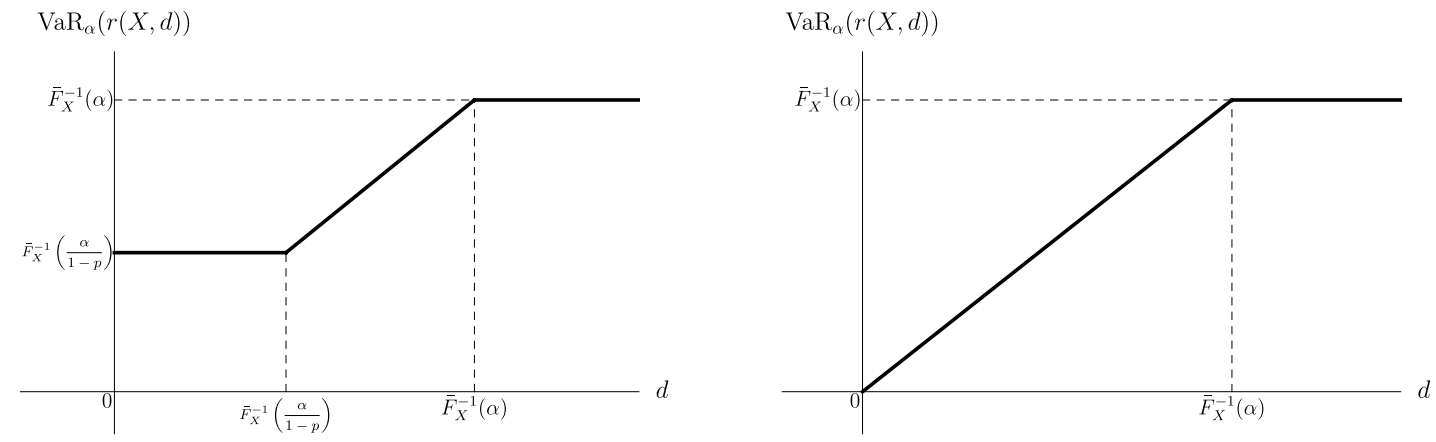

FiguRE 3. $\operatorname{VaR}_{\alpha}(r(X, d))$ as a function of $d$ for $p<1-\alpha / \bar{F}_{X}(0)$ (left) and $p \geq 1-\alpha / \bar{F}_{X}(0)$ (right).

The grey area depicted in Figure 4 represents all additional pairs $\left(d, \operatorname{VaR}_{\alpha}(r(X, d))\right)$ that can be obtained by varying $p$ in the range $p \in\left(0,1-\alpha / \bar{F}_{X}(0)\right)$. 


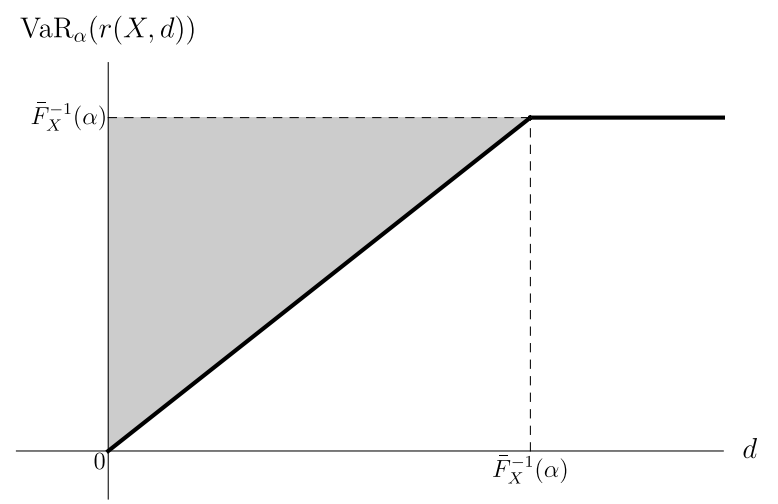

FiguRE 4. Effect of randomizing on the Value-at-Risk as a function of $d$

\subsection{Optimization w.r.t. $d$ for fixed $p$.}

Proposition 4.1. Fix the value of $p$ and let $\kappa:=\frac{1}{p\left(1+\theta / r_{C o C}\right)}$.

(i) Consider first the case $p \geq 1-\alpha / \bar{F}_{X}(0)$. If

$$
\alpha<\kappa<\bar{F}_{X}(0)
$$

and

$$
g\left(\bar{F}_{X}^{-1}(\kappa), p\right) \leq \bar{F}_{X}^{-1}(\alpha),
$$

then a finite optimal retention $d^{*}$ exists and is given by

$$
d^{*}=\bar{F}_{X}^{-1}(\kappa) \text {. }
$$

If

$$
\alpha<\bar{F}_{X}(0) \leq \kappa
$$

and

$$
\mathbb{E}[X] \leq \kappa \bar{F}_{X}^{-1}(\alpha)
$$

hold, then the finite optimal retention is $d^{*}=0$.

(ii) For $p \in\left(0,1-\alpha / \bar{F}_{X}(0)\right)$, a finite optimal retention $d^{*}$ exists if

$$
\alpha<\kappa<\frac{\alpha}{1-p}
$$

and

$$
g\left(\bar{F}_{X}^{-1}(\kappa), p\right) \leq \bar{F}_{X}^{-1}(\alpha)
$$


and then its value is also

$$
d^{*}=\bar{F}_{X}^{-1}(\kappa)
$$

Alternatively, if

$$
\kappa \geq \frac{\alpha}{1-p}
$$

and

$$
g\left(\bar{F}_{X}^{-1}\left(\frac{\alpha}{1-p}\right), p\right) \leq \bar{F}_{X}^{-1}(\alpha),
$$

the optimal retention is given by

$$
d^{*}=\bar{F}_{X}^{-1}\left(\frac{\alpha}{1-p}\right) .
$$

If none of the above conditions hold, $d^{*}=\infty$ (i.e. no reinsurance).

Proof. Let us first consider the case $p \in\left[1-\alpha / \bar{F}_{X}(0), 1\right]$. Then,

$$
g(d):=g(d, p)= \begin{cases}g_{L}(d), & 0 \leq d \leq \bar{F}_{X}^{-1}(\alpha) \\ g_{U}(d), & d>\bar{F}_{X}^{-1}(\alpha)\end{cases}
$$

with

$$
\begin{aligned}
& g_{L}(d):=\left(1+\frac{\theta}{r_{C o C}}\right) p \int_{d}^{\infty} \bar{F}_{X}(x) d x+d, \\
& g_{U}(d):=\left(1+\frac{\theta}{r_{C o C}}\right) p \int_{d}^{\infty} \bar{F}_{X}(x) d x+\bar{F}_{X}^{-1}(\alpha) .
\end{aligned}
$$

Clearly, from $(28), g(d)$ is continuous on $d \in[0, \infty)$ and tends to $\bar{F}_{X}^{-1}(\alpha)$ as $d \rightarrow$ $\infty$. In addition, observe that for $\kappa<\bar{F}_{X}(0), g_{L}(d)$ is decreasing on $\left[0, \bar{F}_{X}^{-1}(\kappa)\right)$, increasing on $\left(\bar{F}_{X}^{-1}(\kappa), \infty\right)$ and attains a minimum at $\bar{F}_{X}^{-1}(\kappa)$. Therefore, since $g_{U}(d)$ is decreasing on $d \in[0, \infty), g(d)$ attains a global minimum at $\bar{F}_{X}^{-1}(\kappa)$ if $\alpha<$ $\kappa<\bar{F}_{X}(0)$ and $g\left(\bar{F}_{X}^{-1}(\kappa)\right) \leq \bar{F}_{X}^{-1}(\alpha)$. Here, the latter condition ensures that a finite global minimum of $g(d)$ exists, namely that the expected profit $\mathbb{E}[Z(d)]$ can be increased through reinsurance. In this case, the optimal retention is $d^{*}=\bar{F}_{X}^{-1}(\kappa)$. The condition $\alpha<\kappa$ is necessary, otherwise $\bar{F}_{X}^{-1}(\kappa) \geq \bar{F}_{X}^{-1}(\alpha)$ and $g(d)$ is then decreasing on $d \in[0, \infty)$ in which case a finite optimal retention $d^{*}$ does not exist (i.e. it would be preferable not to buy reinsurance from a profitability aspect). Note 
that if $\kappa \geq \bar{F}_{X}(0), g_{L}(d)$ attains its minimum at $d=0$ and is increasing on $d \in(0, \infty)$. Consequently, because $g_{U}(d)$ is decreasing on $d \in[0, \infty)$, a finite optimal retention exists if and only if $g(0)=\left(1+\frac{\theta}{r_{C o C}}\right) p \mathbb{E}[X] \leq \bar{F}_{X}^{-1}(\alpha)$ which can be rewritten as $\mathbb{E}[X] \leq \kappa \bar{F}_{X}^{-1}(\alpha)$. Hence, in this case, the optimal retention is $d^{*}=0$ meaning that the expected profit is maximized by passing the entire risk to the reinsurer.

Let us now examine the case $p \in\left(0,1-\frac{\alpha}{\bar{F}_{X}(0)}\right)$, where

$$
g(d)= \begin{cases}\left(1+\frac{\theta}{r_{C o C}}\right) p \int_{d}^{\infty} \bar{F}_{X}(x) d x+\bar{F}_{X}^{-1}\left(\frac{\alpha}{1-p}\right), & 0 \leq d<\bar{F}_{X}^{-1}\left(\frac{\alpha}{1-p}\right) \\ \left(1+\frac{\theta}{r_{C o C}}\right) p \int_{d}^{\infty} \bar{F}_{X}(x) d x+d, & \bar{F}_{X}^{-1}\left(\frac{\alpha}{1-p}\right) \leq d \leq \bar{F}_{X}^{-1}(\alpha), \\ \left(1+\frac{\theta}{r_{C o C}}\right) p \int_{d}^{\infty} \bar{F}_{X}(x) d x+\bar{F}_{X}^{-1}(\alpha), & d>\bar{F}_{X}^{-1}(\alpha) .\end{cases}
$$

Again, $g(d)$ is continuous on $d \in[0, \infty)$ with the limiting value $\bar{F}_{X}^{-1}(\alpha)$ as $d \rightarrow \infty$. Furthermore, observe from $(29)$ that $g(d)$ is decreasing on $d \in\left[0, \bar{F}_{X}^{-1}\left(\frac{\alpha}{1-p}\right)\right)$. The subsequent behavior of $g(d)$ is determined by the relations between $\kappa, \alpha$ and $p$. More precisely, if $\kappa \geq \frac{\alpha}{1-p}, g_{L}(d)$ is increasing on $d \in\left(\bar{F}_{X}^{-1}\left(\frac{\alpha}{1-p}\right), \infty\right)$, so is $g(d)$ on $d \in\left(\bar{F}_{X}^{-1}\left(\frac{\alpha}{1-p}\right), \bar{F}_{X}^{-1}(\alpha)\right]$. Hence, because $g_{U}(d)$ is decreasing on $d \in[0, \infty)$, a finite optimal retention $d^{*}$ exists only if $g\left(\bar{F}_{X}^{-1}\left(\frac{\alpha}{1-p}\right)\right) \leq \bar{F}_{X}^{-1}(\alpha)$. In this case, $g(d)$ attains a global minimum at $\bar{F}_{X}^{-1}\left(\frac{\alpha}{1-p}\right)$, which is the optimal retention. In the case $\alpha<\kappa<\frac{\alpha}{1-p}, g(d)$ is decreasing on $d \in\left[0, \bar{F}_{X}^{-1}(\kappa)\right)$, increasing on $\left(\bar{F}_{X}^{-1}(\kappa), \bar{F}_{X}^{-1}(\alpha)\right]$ and then decreasing again towards $\bar{F}_{X}^{-1}(\alpha)$ as $d \rightarrow \infty$. The function $g(d)$ then attains a global minimum value at $\bar{F}_{X}^{-1}(\kappa)$ if $g\left(\bar{F}_{X}^{-1}(\kappa)\right) \leq \bar{F}_{X}^{-1}(\alpha)$. Hence, $d^{*}=$ $\bar{F}_{X}^{-1}(\kappa)$ is the optimal retention. Finally, if $\kappa \leq \alpha, f(d)$ is decreasing on $d \in[0, \infty)$. Consequently, a finite optimal retention $d^{*}$ does not exist.

\section{Numerical ILlustrations}

In this section, we illustrate the effects of the proposed randomized stop-loss treaty on the expected profit and discuss some quantitative properties of the resulting optimal retention level $d^{*}$. Assume that the distribution function of the aggregate loss of the insurer is given by

$$
F_{X}(x)= \begin{cases}0.05, & x=0 \\ 1-0.95\left(\frac{1000}{1000+x}\right)^{3}, & x>0\end{cases}
$$


i.e. a shifted Pareto distribution with an atom at 0. Furthermore, assume that the first-line insurance premium is determined by $\pi(X)=(1+0.1) \cdot \mathbb{E}[X]=522.5$.

5.1. Optimal retention level $d^{*}$ as a function of $p$. Figure 5 depicts the optimal retention $d^{*}$ as a function of $p$ for $\theta=0.2, r_{C o C}=0.07$ and $\alpha=0.05$. Recall from Section 4.1 that one needs to distinguish the regions $p \in(0, a)$ and $p \in[a, 1]$ with $a:=1-\alpha / \bar{F}_{X}(0) \approx 0.947$ for the analysis (indicated by the right vertical dashed line). In both cases, the existence and representation of the optimal retention $d^{*}$ is contingent on the value of $\kappa=\kappa(p) \approx \frac{0.259}{p}$. For $p \in(0, a)$, the two subcases $\kappa \geq \frac{\alpha}{1-p}$ and $\alpha<\kappa<\frac{\alpha}{1-p}$ have to be treated separately (see the left vertical dashed line at $p \approx 0.838$ for which $\left.\kappa=\frac{\alpha}{1-p}\right)$. In all these cases, the conditions of Proposition 4.1 are verified for the considered parameter set and any $p \in(0,1]$, so that a finite optimal retention level $d^{*}$ is known to exist. For $p \in(0,0.838]$ (i.e., $\left.\kappa \geq \frac{\alpha}{1-p}\right)$, the optimal retention $d^{*}$ is given by $\bar{F}_{X}^{-1}\left(\frac{\alpha}{1-p}\right)$ and is decreasing in $p$. In this region, in order to maximize the expected profit, it is optimal to choose $d$ such that the VaR is minimized; the gains from a cheaper reinsurance premium with a larger retention would not offset the additional costs arising from a larger VaR. As $p$ increases within this region, smaller VaR values can be attained (cf. Figure 4), explaining the decrease in the optimal retention $d^{*}$ up to $p=0.838$. The rate of this decrease corresponds to the rate at which the VaR domain is enlarged (as a function of $d$ ) when $p$ increases. At $p=0.838$, the savings on the reinsurance premium from choosing larger values of $d$ start to dominate the capital costs for resulting higher VaR values. As a result, the optimal retention given by $d^{*}=\bar{F}_{X}^{-1}(\kappa)$ increases on $p \in(0.838,1]$ with a smooth transition through the right vertical line at $p=0.947$.

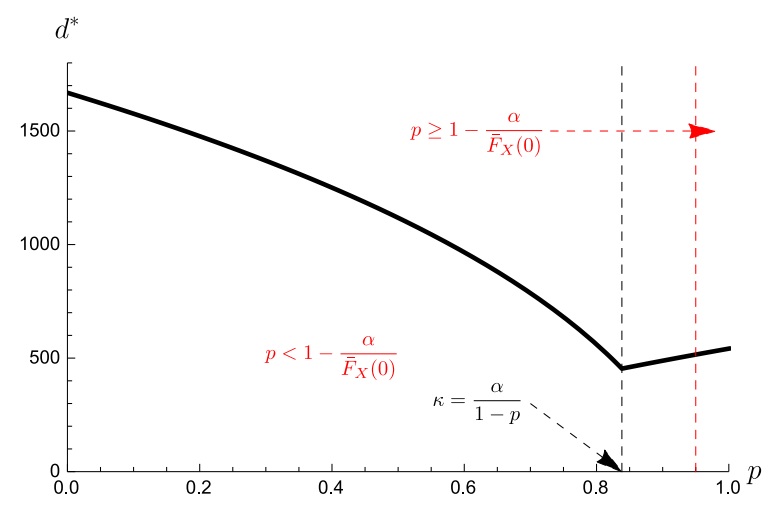

Figure 5. Optimal retention $d^{*}$ as a function of $p$ for $\theta=0.2$ (solid) with $\alpha=0.05$ and $r_{C o C}=0.07$. 
Let us now examine the effects of the reinsurance loading $\theta$ on the optimal retention level $d^{*}$. When reinsurance becomes cheaper, i.e. $\theta$ decreases, reinsurance premium savings are reduced. This has the effect of shifting the solution $p$ of $\kappa(p)=\frac{\alpha}{1-p}$ towards higher $p$-values, in the present case to 0.864 for $\theta=0.15$ and 0.886 for $\theta=0.11$. Since reinsurance premium savings become worth considering only for higher $p$-values, minimizing the $\mathrm{VaR}$ is of interest in an extended region, resulting in an extended decrease of $d^{*}$ for smaller reinsurance loadings (cf. Figure 6).

Note that $\theta$ and $r_{C o C}$ enter in $\kappa$ as a ratio, so if both these parameters increase or decrease to the same relative extent, the resulting shape of the optimal retention $d^{*}$ as a function of $p$ will remain unchanged.

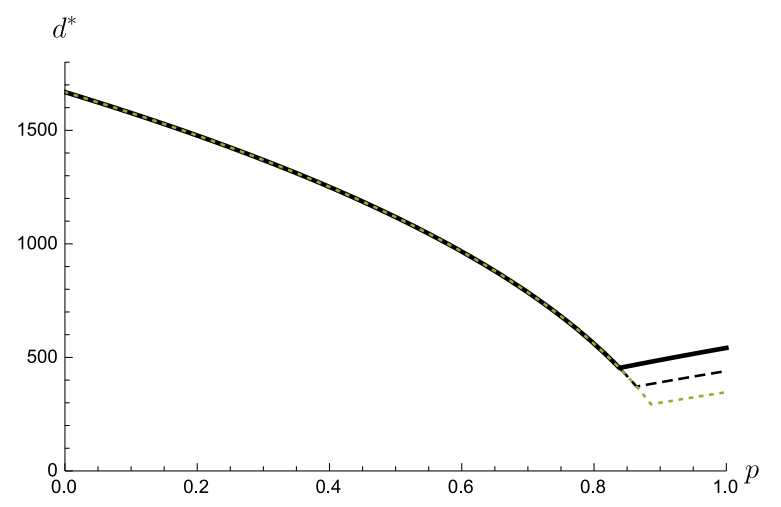

Figure 6. Optimal retention $d^{*}$ as a function of $p$ for $\theta=0.11$ (dotted), $\theta=0.15$ (dashed) and $\theta=0.2$ (solid) with $\alpha=0.05$ and $r_{C o C}=0.07$.

Another observation is that the optimal retention $d^{*}$ is not affected by a change in the reinsurance premium loading up to $p=0.838$ for the considered $\theta$-values (here, $\theta$ almost doubles form 0.11 to 0.2 ). The reason is again the trade-off between VaR and reinsurance premium (and the fact the reinsurance premium is based on the expected value principle). In other words, if $p$ is fixed at such (not too large) values, an increased reinsurance premium will still lead to the same insurer's preference choice of the retention. In order to further illustrate this point, Figure 7 depicts $d^{*}$ as a function of $\theta$ for a fixed value of $p=0.8$. For all values of $\theta$ up to $\theta=0.28$ (which signifies the value for which $\kappa(\theta)=\frac{\alpha}{1-p}$ ), $d^{*}$ remains unchanged. Beyond that value, $d^{*}$ increases. Finally, for $\theta \geq 0.449$ condition (25) is not fulfilled any more, and reinsurance becomes too expensive for the insurer to enter a reinsurance agreement of this type at all.

5.2. Optimal $p^{*}$ as a function of the retention $d$. For each given retention level $d \geq 0$, one can also look for the optimal $p \in[0,1]$ that maximizes the expected profit. 


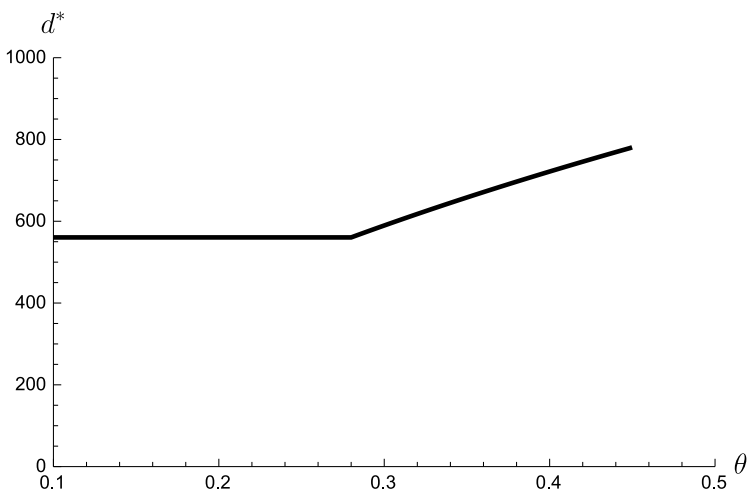

Figure 7. Optimal retention $d^{*}$ as a function of $\theta$ for $p=0.8$.

Figure 8 depicts $p^{*}$ as a function of the retention level $d$ for different reinsurance loadings. Note that for small values of $d$ and high reinsurance loading, it is preferable to have no reinsurance at all.

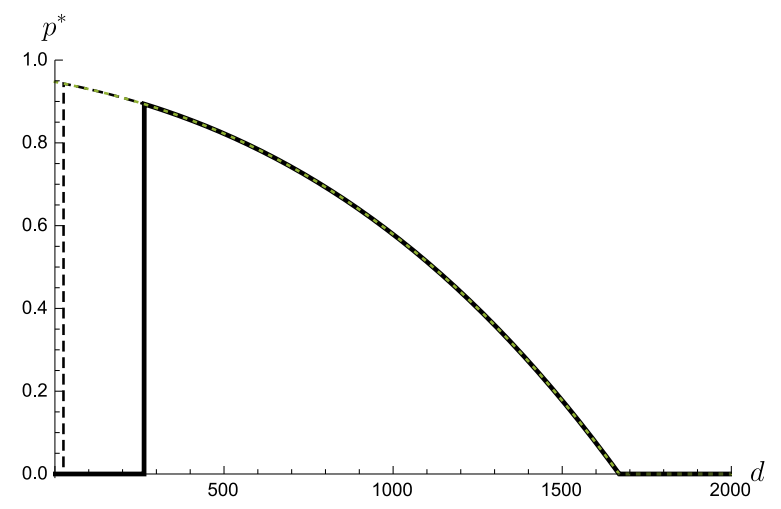

Figure 8. Optimal $p^{*}$ as a function of $d$ for $\theta=0.11$ (dotted), $\theta=0.2$ (dashed) and $\theta=0.3$ (solid).

5.3. Maximal expected profit as a function of $p$. Let us now analyze the impact of introducing randomness in the reinsurance treaty on the expected profit. Figure 9 depicts the maximal expected profit (under the choice of the respective best $d^{*}(p)$ ) as a function of $p$ for various reinsurance loadings. It is interesting to observe that although $d^{*}$ is first decreasing in $p$ on $\kappa \geq \frac{\alpha}{1-p}$, the expected profit is first increasing in $p$. Thus, having the possibility to choose a smaller VaR (by decreasing $d^{*}$ ) in response to an increase in $p$ outbalances the increase in the reinsurance premium (through both an increase of $p$ and decrease of $d^{*}$ ) in an increasing fashion. The maximal expected profit is attained when the optimal pair $\left(d^{*}, p^{*}\right)$ is chosen. In the 
present illustration $p^{*}$ is 0.863 for $\theta=0.11,0.829$ for $\theta=0.15$ and 0.804 for $\theta=0.18$. As $p$ approaches 1 , the gains diminish again. Note that the randomized strategy outperforms the classical deterministic stop-loss $(p=1)$ for a variety of $p$-values. One also sees that for higher reinsurance premiums (here $\theta=0.15$ and $\theta=0.18$ ), an over-all positive expected profit can only be achieved through randomization, not with a determinstic stop-loss contract (even when using the optimal retention). Figure 10 depicts the expected profit for arbitrary combinations of retentions $d$ and probabilities $p$.

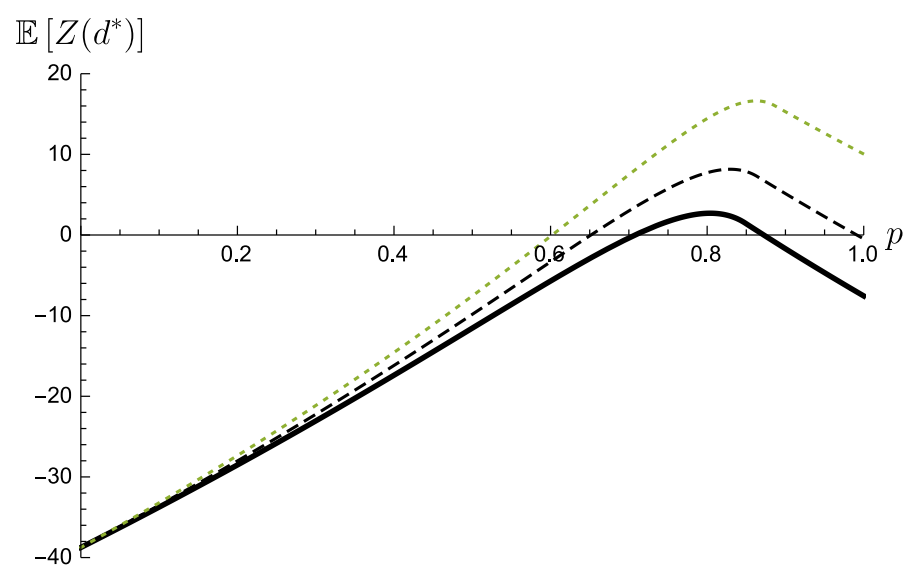

Figure 9. Maximal expected profit $\mathbb{E}\left[Z\left(d^{*}\right)\right]$ as a function of $p$ for $\theta=0.11$ (dotted), $\theta=0.15$ (dashed) and $\theta=0.18$ (black) with $\alpha=$ 0.05 and $r_{C o C}=0.07$.

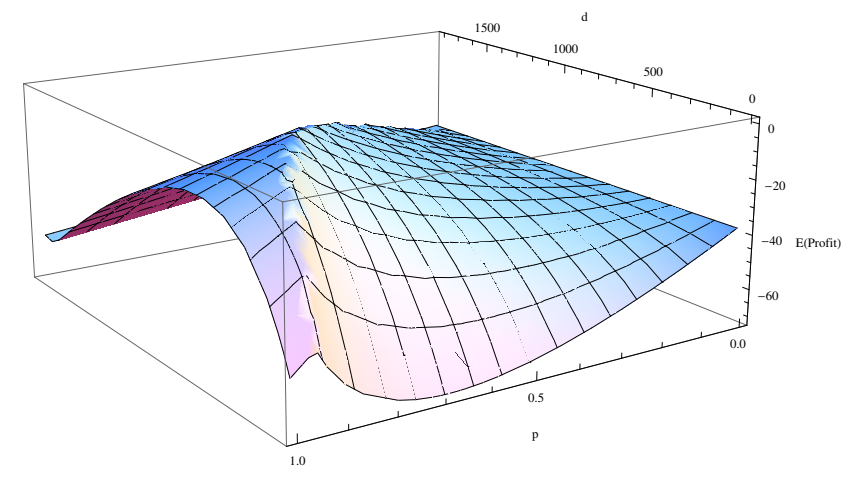

Figure 10. Expected profit as a function of $d$ and $p$. 


\section{Comparison With BOUNDED STOP-LOSS CONTRACTS}

Randomization adds a degree of freedom to the classical stop-loss treaty, and so one may argue that this naturally leads to an improved solution. From Figure 1 it becomes clear that the resulting shape of the retained loss distribution of a randomized stop-loss treaty resembles a deterministic bounded stop-loss treaty

$$
r_{B}(X)=x-\min \left\{\left(X-d_{B}\right)_{+}, l_{B}\right\}
$$

with retention $d_{B}$ and upper limit $l_{B}$. It is hence particularly instructive to compare the two. Note that beyond the retention the former takes a convex combination of the original loss c.d.f. $F_{X}$ and the constant 1, whereas the latter shifts the part of $F_{X}$ to the right of $d_{B}+l_{B}$ by $l_{B}$ units to the left, cf. Figure 11. Hence, even for $d=d_{B}$, the resulting contracts will in general be different.
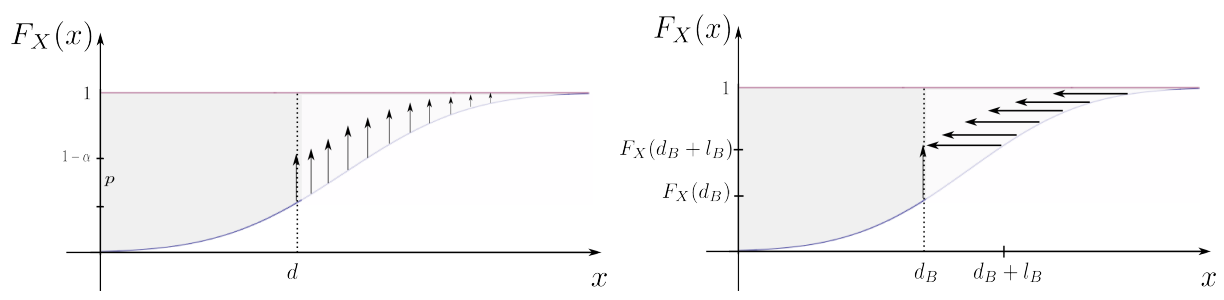

Figure 11. Original and retained loss distribution under randomized reinsurance (1) (left) and the bounded stop-loss contract (30) (right)

In [14], it has been shown that a bounded stop-loss treaty minimizes the total retained risk exposure of an insurer within the class of deterministic reinsurance forms where both the ceded and retained loss functions are non-decreasing. As outlined in Remark 2.1, under the expected value principle for the reinsurance premium, this then also applies to the objective function used in the present paper, but under different weights for the sum of the competing terms. We now want to compare the optimal randomized strategy $\left(d^{*}, p^{*}\right)$ with the optimal bounded stop-loss treaty $\left(d_{B}^{*}, l_{B}^{*}\right)$. By similar arguments as in Section 3 (or also following the reasoning in $[14])$, it is clear that it is better not to take any reinsurance if $d_{B}^{*} \geq \bar{F}_{X}^{-1}(\alpha)$, and in the other case necessarily $l_{B}^{*}=l_{B}^{*}\left(d_{B}^{*}\right)=\bar{F}_{X}^{-1}(\alpha)-d_{B}^{*}$. That is,

$$
r_{B}^{*}(x)= \begin{cases}x-\min \left(\left(x-d_{B}^{*}\right)_{+}, \bar{F}_{X}^{-1}(\alpha)-d_{B}^{*}\right), & \text { if } d_{B}^{*}<\bar{F}_{X}^{-1}(\alpha), \\ x, & \text { if } d_{B}^{*} \geq \bar{F}_{X}^{-1}(\alpha),\end{cases}
$$


with $d_{B}^{*}=\bar{F}_{X}^{-1}\left(\frac{1}{1+\theta / r_{C o C}}\right)$. The analogue of (8) for the optimal bounded stop-loss then is

$$
g_{B}\left(d_{B}^{*}\right):= \begin{cases}\left(1+\frac{\theta}{r_{C o C}}\right) \int_{d_{B}^{*}}^{\bar{F}_{X}^{-1}(\alpha)} \bar{F}_{X}(x) d x+d_{B}^{*}, & \text { if } d_{B}^{*}<\bar{F}_{X}^{-1}(\alpha), \\ \bar{F}_{X}^{-1}(\alpha), & \text { if } d_{B}^{*} \geq \bar{F}_{X}^{-1}(\alpha) .\end{cases}
$$

For the best randomized stop-loss treaty, the respective amount reads

$g\left(d^{*}, 1-\frac{\alpha}{\bar{F}_{X}\left(d^{*}\right)}\right)= \begin{cases}\left(1+\frac{\theta}{r_{C o C}}\right)\left(1-\frac{\alpha}{\bar{F}_{X}\left(d^{*}\right)}\right) \int_{d^{*}}^{\infty} \bar{F}_{X}(x) d x+d^{*}, & \text { if } d^{*}<\bar{F}_{X}^{-1}(\alpha), \\ \bar{F}_{X}^{-1}(\alpha), & \text { if } d^{*} \geq \bar{F}_{X}^{-1}(\alpha),\end{cases}$

where $d^{*}$ is determined according to Section 3 .

Let us consider any candidate retention $0 \leq d<\bar{F}_{X}^{-1}(\alpha)$ and $d=d_{B}$. Then for both the randomized stop-loss and the bounded stop-loss, the choice $p^{*}(d)$ and $l_{B}^{*}(d)$ will be such that the resulting $\operatorname{VaR}_{\alpha}(r(X))$ is equal to $d$. To quantify the performance difference of the two treaties one is thus left with comparing the pure reinsurance premiums:

$$
\begin{aligned}
h(d): & =\left(1-\frac{\alpha}{\bar{F}_{X}(d)}\right) \int_{d}^{\infty} \bar{F}_{X}(x) d x-\int_{d}^{\bar{F}_{X}^{-1}(\alpha)} \bar{F}_{X}(x) d x \\
& =\int_{\bar{F}_{X}^{-1}(\alpha)}^{\infty} \bar{F}_{X}(x) d x-\alpha \cdot e_{X}(d), \\
& =\alpha\left(e_{X}\left(\bar{F}_{X}^{-1}(\alpha)\right)-e_{X}(d)\right) .
\end{aligned}
$$

Correspondingly, if the mean-excess function is increasing, which is a property typically shared by the class of heavy-tailed distributions (see e.g. Embrechts et al. [17, Ch.6]), it follows that a bounded stop-loss treaty is preferable to a randomized stop-loss treaty for each fixed retention level $d=d_{B}$. In other words, shifting the distribution by $l_{B}^{*}(d)$ to obtain $\operatorname{VaR}_{\alpha}(r(X))=d$ then leads to a cheaper premium than reshaping the c.d.f. by randomization towards $\operatorname{VaR}_{\alpha}(r(X))=d$. Since this is true for all $d$, the best bounded stop-loss treaty then also outperforms the best randomized stop-loss treaty.

On the other hand, for distributions with decreasing mean-excess function (like the uniform distribution, certain Gamma distributions or the light-tailed Weibull distribution), randomization outperforms bounded stop-loss for each retention level and correspondingly also for the respective optimal retention levels.

When the mean-excess function is not monotone, the performance comparison can be more intricate, cf. Example 6.4. 
In the following, we consider some concrete examples.

Example 6.1. If $X$ is exponentially distributed, the mean-excess function $e_{X}(d)$ is constant, so that $h(d)=0$ for all $d<\bar{F}_{X}^{-1}(\alpha)$. This means that in this case the best randomized stop-loss and the best bounded stop-loss treaty lead to the same resulting loss distribution, and correspondingly the optimal values $d^{*}$ and $d_{B}^{*}$ must coincide. This is of course due to the lack-of-memory property of the exponential distribution: for the region to the right of the retention level $d$, shifting the distribution function from the right by $l_{B}^{*}(d)$ into the point $(d, 1-\alpha)$ is equivalent to rescaling it up into that same attachment point. This can also easily be verified analytically by realizing that $\mathbb{P}(r(X, d)>d+y)=\alpha \mathbb{P}(X>y)$ as well as $\mathbb{P}\left(r_{B}(X)>d_{B}+y\right)=\alpha \mathbb{P}(X>y)$ for the respective optimal values $p^{*}(d)$ and $l_{B}^{*}\left(d_{B}\right)$ and all $y>0$.

Example 6.2. Let $X$ be uniformly distributed in $[0, b]$, in which case $\bar{F}_{X}^{-1}(\alpha)=$ $b(1-\alpha)$. Here $e_{X}(d)$ is decreasing in $d$, so a randomized stop-loss will lead to a better profitability. The optimal bounded stop-loss is the following: If $1 / \alpha>1+$ $\theta / r_{C o C}$, then the retention $d_{B}^{*}=b\left(1-\frac{1}{1+\frac{\theta}{r_{C o C}}}\right)$ is chosen together with the layer $l_{B}^{*}\left(d_{B}^{*}\right)=b\left(\frac{1}{1+\frac{\theta}{r_{C o C}}}-\alpha\right)$, otherwise it is preferable not to buy reinsurance. After some calculations, one gets

$$
g_{B}\left(d_{B}^{*}\right)= \begin{cases}\frac{b}{2}\left(-\alpha^{2}\left(1+\frac{\theta}{r_{C o C}}\right)+\frac{1+\frac{2 \theta}{r_{C O C}}}{1+\frac{\theta}{r_{C o C}}}\right), & \text { if } \frac{1}{\alpha}>1+\frac{\theta}{r_{C o C}}, \\ b(1-\alpha), & \text { if } \frac{1}{\alpha} \leq 1+\frac{\theta}{r_{C o C}} .\end{cases}
$$

At the same time, under the optimal randomized stop-loss, we have in view of (15)

$$
g\left(d^{*}, p^{*}\right)= \begin{cases}b\left(1-\frac{1}{2}\left(\alpha+\frac{1}{1+\frac{\theta}{r_{C o C}}}\right)-\frac{1}{8} \alpha^{2}\left(1+\frac{\theta}{r_{C o C}}\right)\right), & \text { if } \frac{2}{\alpha}>1+\frac{\theta}{r_{C o C}}, \\ b(1-\alpha), & \text { if } \frac{2}{\alpha} \leq 1+\frac{\theta}{r_{C o C}} .\end{cases}
$$

The difference $g_{B}\left(d_{B}^{*}\right)-g\left(d^{*}, p^{*}\right):=D$ reads

$$
D= \begin{cases}0, & \text { if } \frac{1}{\alpha} \leq \frac{1}{2}\left(1+\frac{\theta}{r_{C o C}}\right), \\ \frac{b}{2}\left(\frac{1}{1+\frac{\theta}{r_{C o C}}}+\alpha\left(\frac{\alpha}{2}\left(1+\frac{\theta}{r_{C o C}}\right)-1\right)\right)>0, & \text { if } \frac{1}{2}\left(1+\frac{\theta}{r_{C o C}}\right)<\frac{1}{\alpha} \leq\left(1+\frac{\theta}{r_{C o C}}\right), \\ \frac{b \alpha}{2}\left(1-\frac{3}{4} \alpha\left(1+\frac{\theta}{r_{C o C}}\right)\right)>0, & \text { if } \frac{1}{\alpha}>1+\frac{\theta}{r_{C o C}} .\end{cases}
$$


Correspondingly, the best randomized stop-loss treaty is always at least as good as the best bounded stop-loss contract, and typically better. Note that the performance difference increases in $b$. It is also worth mentioning that for a uniformly distributed risk with a bounded stop-loss, the c.d.f. of the retained amount attains 1 at $b-l_{B}^{*}<b$, which is sub-optimal in view of minimizing the reinsurance premium. Conversely, by construction, the resulting c.d.f. of randomized stop-loss attains 1 only at b. Figure 12 illustrates the expected profit under the optimal bounded stop-loss (dashed) and the optimal randomized stop-loss (solid) for $\alpha=0.05, r_{\mathrm{CoC}}=0.07$ and $b=5$ as a function of the premium loading $\theta$.

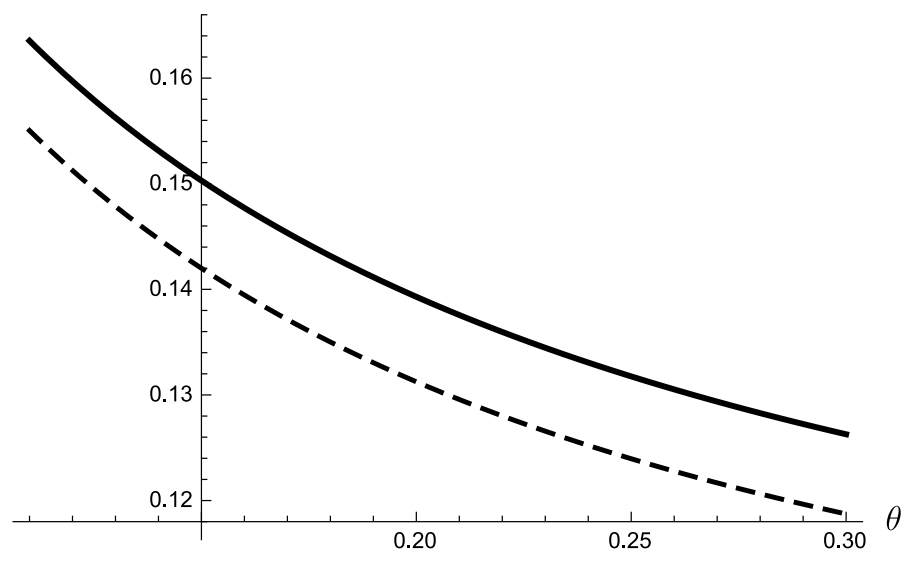

Figure 12. Expected profit with the optimal bounded stop-loss (dashed) vs. optimal randomized stop-loss (solid)

Example 6.3. Let $X$ be a shifted Pareto random variable with

$$
F_{X}(x)=1-\left(\frac{\xi}{x+\xi}\right)^{1 / \gamma}, \quad \xi>0 ; \gamma<1
$$

In this case the mean-excess function $e_{X}(d)$ is increasing, so a randomized stop-loss treaty can not outperform the best bounded stop-loss. The optimal bounded stoploss strategy is as follows: If $1 / \alpha>1+\theta / r_{C o C}$, then one chooses the retention $d_{B}^{*}=$ 
$\xi\left(\left(\frac{1}{1+\frac{\theta}{r_{C o C}}}\right)^{-\gamma}-1\right)$ together with the layer $l_{B}^{*}\left(d_{B}^{*}\right)=\xi\left(\alpha^{-\gamma}-\left(\frac{1}{1+\frac{\theta}{r_{C o C}}}\right)^{-\gamma}\right)$, otherwise no reinsurance is taken. In view of (32), this translates into

$g_{B}\left(d_{B}^{*}\right):= \begin{cases}\xi\left(\left(\frac{1}{1+\frac{\theta}{r_{C o C}}}\right)^{-\gamma}-1+\frac{\left(1+\frac{\theta}{r_{C o C}}\right)}{\frac{1}{\gamma}-1}\left(\left(\frac{1}{1+\frac{\theta}{r_{C o C}}}\right)^{1-\gamma}-\alpha^{1-\gamma}\right)\right), & \text { if } \frac{1}{\alpha}>1+\frac{\theta}{r_{C o C}}, \\ \xi\left(\alpha^{-\gamma}-1\right), & \text { if } \frac{1}{\alpha} \leq 1+\frac{\theta}{r_{C o C}} .\end{cases}$

On the other hand, in view of (14), the optimal randomized stop-loss strategy is given by

$$
g\left(d^{*}, p^{*}\right)= \begin{cases}\xi\left(\left(\frac{1}{a}-\frac{\alpha}{\frac{1}{\gamma}-1}\right)^{-\gamma}\left(1+\frac{a}{\frac{1}{\gamma}-1}\left(\frac{1}{a}-\frac{\alpha}{1-\gamma}\right)\right)-1\right), & \text { if } \frac{1}{\alpha}(1-\gamma)>a \\ \xi\left(\alpha^{-\gamma}-1\right), & \text { if } \frac{1}{\alpha}(1-\gamma) \leq a\end{cases}
$$

where $a:=1+\frac{\theta}{r_{C o C}}$. The difference $D:=g_{B}\left(d_{B}^{*}\right)-g\left(d^{*}, 1-\frac{\alpha}{F_{X}\left(d^{*}\right)}\right)$ then takes the form

$$
D= \begin{cases}0, & \text { if } \frac{1}{\alpha} \leq a, \\ \xi\left(a^{\gamma}-\alpha^{-\gamma}+\frac{a}{\frac{1}{\gamma}-1}\left(a^{\gamma-1}-\alpha^{1-\gamma}\right)\right)<0, & \text { if } a<\frac{1}{\alpha} \leq \frac{a}{1-\gamma}, \\ \frac{\xi\left(\left(\frac{1}{\gamma}-1\right)\left(\frac{a^{\gamma}}{\gamma}-a \alpha^{1-\gamma}\right)+\frac{1}{\gamma}\left(1+a \alpha-\frac{1}{\gamma}\right)\left(\frac{1}{a}-\frac{\alpha}{\frac{1}{\gamma}-1}\right)^{-\gamma}\right)}{\left(\frac{1}{\gamma}-1\right)^{2}}<0, & \text { if } \frac{1}{\alpha}>\frac{a}{1-\gamma},\end{cases}
$$

so that indeed here a bounded stop-loss contract is always preferable.

Example 6.4. Let us now consider an example of a distribution with non-monotone mean-excess function. Concretely, let us introduce an upper truncation point $T>0$ to the shifted Pareto distribution considered in Example 6.3, i.e.

$$
F_{X}(x)=\frac{1-\left(\frac{\xi}{x+\xi}\right)^{\frac{1}{\gamma}}}{1-\left(\frac{\xi}{T+\xi}\right)^{\frac{1}{\gamma}}}, \quad 0 \leq x \leq T ; \xi>0 ; \gamma<1 .
$$

Such distributions recently gained some popularity in insurance claims modelling (see e.g. [1, Ch.4]). The corresponding mean-excess function is

$$
e_{X}(d)=\frac{\left(\frac{\xi}{T+\xi}\right)^{\frac{1}{\gamma}}\left(d+\xi+\frac{(T-d)}{\gamma}\right)-\left(\frac{\xi}{d+\xi}\right)^{\frac{1}{\gamma}}(d+\xi)}{\left(\frac{1}{\gamma}-1\right)\left(\left(\frac{\xi}{T+\xi}\right)^{\frac{1}{\gamma}}-\left(\frac{\xi}{d+\xi}\right)^{\frac{1}{\gamma}}\right)}, \quad 0 \leq d<T
$$


which is non-monotone (first increasing and then decreasing to 0) for triples $(\xi, \gamma, T)$ with $e_{X}^{\prime}(0)>0$ (cf. Figure 13).

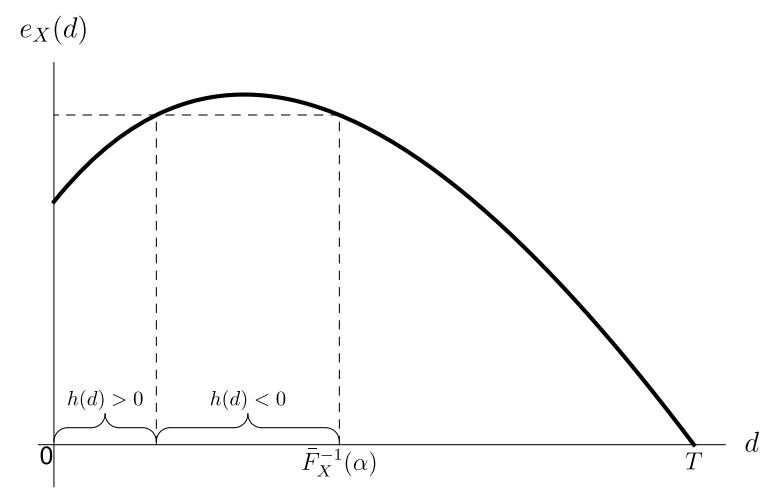

Figure 13. Possible shape of the mean-excess function for a shifted truncated Pareto random variable

Figure 14 depicts the expected profit under both treaties as a function of the truncation point $T$ for $\xi=20, \gamma=0.5, \alpha=0.05, \theta=0.2$ and $r_{C o C}=0.07$. One sees that there is a threshold value for $T$ above which the heavy-tailed feature of the risk $X$ starts to dominate, making bounded stop-loss more attractive. However, for smaller values of $T$ the randomized stop-loss is preferable.

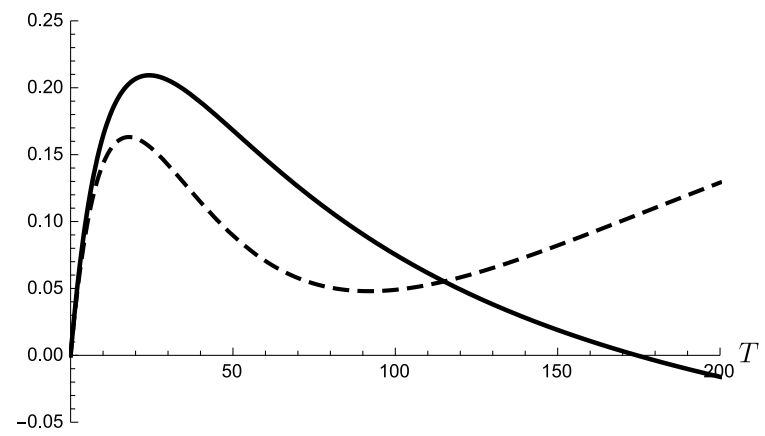

FiguRE 14. Expected profit with optimal bounded stop-loss (dashed) vs. optimal randomized stop-loss (solid) as a function of $T$

\section{Conclusion}

In this paper, we showed that randomizing classical reinsurance treaties can be beneficial for the insurer. While randomization is a well-known mathematical tool to help 
identifying optimal deterministic solutions, the purpose here was to initiate a discussion and ponder the possibilities of actually implementing additional randomness in the settlement of the risk sharing arrangement between insurer and reinsurer. In this context, one should keep in mind that randomization has potential advantages with respect to moral hazard problems, as it is unclear during the settlement procedure who will finally have to pay the claim. Also, when comparing the randomized stoploss treaty with deterministic bounded stop-loss, one may argue that in the former case the resulting retained loss distribution beyond the retention is determined by a part closer to the center for which one may have more confidence in the chosen model (as in the latter the respective part is further in the tail of the original loss distribution).

We deliberately chose a simple form of randomization as well as simple model assumptions here, in order to make the reasoning transparent, and clearly many variants and generalizations are possible. This includes considering more general reinsurance premium principles, but also randomization of individual claims (like in excess-of-loss treaties). For instance, rather than participating with a fraction $p$ in all claims like in a quota-share arrangement, the reinsurer could achieve a similar result by paying each claim fully, but only with a probability $p$, independently for each claim (which can be preferable in terms of administrative expenses). On the aggregate level, one can view the introduced randomization also as a simple alternative way to reshape the loss distribution (for instance when 'picking' any target point above the original loss distribution function for the retained loss distribution function, one can realize the resulting risk transfer through simple randomization. This can in general be a simple means to taylor the needs of clients for reinsurance companies (in terms of target shapes of the retained loss), and more intricate randomization mechanisms can further increase the possible variations. While the concept can seem non-intuitive in the first place, it may provide a thought-provoking additional perspective on the nature of the problem (as well as on the choice of objective functions and constraints). In this paper, we focused on the Value-at-Risk for measuring risk, and the results depend crucially on this choice. In a subsequent study, we will consider the effects of randomization for other choices of risk measures. However, the Value-at-Risk is the risk measure implemented in many regulatory systems nowadays, and the arguments in this paper may underline some of the shortfalls of this risk measure (particularly the encouragement to 'only' optimize the retained situation up to the point of the solvency requirement). Clearly, in practical situations the solvency ratio will often be considerably larger than 1 (i.e. the actual capital level will be above the minimum regulatory requirement), and corresponding adaptations of the arguments can then be made.

Finally, in this paper we considered the reinsurer's preferences solely through the 
reinsurance premium rule. It will be interesting future work to include the reinsurer's viewpoint on the suitability of randomized contracts by considering joint optimization criteria.

Acknowledgements. We would like to thank Hans Bühlmann, Damir Filipovic, Stefan Jaschke and Léonard Vincent for stimulating discussions on the topic.

\section{REFERENCES}

[1] H. Albrecher, J. Beirlant, and J. Teugels. Reinsurance: Actuarial and Statistical Aspects. John Wiley \& Sons, Chichester, 2017.

[2] K. J. Arrow. Uncertainty and the welfare economics of medical care. The American Economic Review, 53(5):941-973, 1963.

[3] S. Basak and A. Shapiro. Value-at-risk-based risk management: optimal policies and asset prices. The Review of Financial Studies, 14(2):371-405, 2001.

[4] C. Bernard and W. Tian. Optimal reinsurance arrangements under tail risk measures. Journal of Risk and Insurance, 76(3):709-725, 2009.

[5] T. J. Boonen, K. S. Tan, and S. C. Zhuang. Pricing in reinsurance bargaining with comonotonic additive utility functions. ASTIN Bull., 46(2):507-530, 2016.

[6] K. Borch. An attempt to determine the optimum amount of stop loss reinsurance. Transactions of the 16th International Congress of Actuaries, I(3):597-610, 1960.

[7] J. Cai, C. Lemieux, and F. Liu. Optimal reinsurance with regulatory initial capital and default risk. Insurance: Math. Econom., 57:13-24, 2014.

[8] J. Cai and K. S. Tan. Optimal retention for a stop-loss reinsurance under the VaR and CTE risk measures. Astin Bull., 37(1):93-112, 2007.

[9] J. Cai, K. S. Tan, C. Weng, and Y. Zhang. Optimal reinsurance under VaR and CTE risk measures. Insurance: Math. Econom., 43(1):185-196, 2008.

[10] K. C. Cheung. Optimal reinsurance revisited - a geometric approach. Astin Bull., 40(1):221239, 2010.

[11] Y. Chi. Optimal reinsurance under variance related premium principles. Insurance: Math. Econom., 51(2):310-321, 2012.

[12] Y. Chi. Reinsurance arrangements minimizing the risk-adjusted value of an insurer's liability. ASTIN Bull., 42(2):529-557, 2012.

[13] Y. Chi, X. S. Lin, and K. S. Tan. Optimal reinsurance under the risk-adjusted value of an insurer's liability and an economic reinsurance premium principle. North American Actuarial Journal, pages 1-16, 2017.

[14] Y. Chi and K. S. Tan. Optimal reinsurance under VaR and CVaR risk measures: a simplified approach. Astin Bull., 41(2):487-509, 2011.

[15] Y. Chi and K. S. Tan. Optimal reinsurance with general premium principles. Insurance: Math. Econom., 52(2):180-189, 2013.

[16] C. L. Culp. Structured Finance and Insurance: The ART of Managing Capital and Risk. John Wiley \& Sons, 2011.

[17] P. Embrechts, C. Klüppelberg, and T. Mikosch. Modelling Extremal Events. Springer, Berlin, 1997.

[18] L. Gajek and D. Zagrodny. Optimal reinsurance under general risk measures. Insurance: Math. Econom., 34(2):227-240, 2004. 
[19] L. Gajek and D. Zagrodny. Reinsurance arrangements maximizing insurer's survival probability. Journal of Risk and Insurance, 71(3):421-435, 2004.

[20] M. Guerra and M. d. L. Centeno. Optimal reinsurance policy: The adjustment coefficient and the expected utility criteria. Insurance: Math. Econom., 42(2):529-539, 2008.

[21] M. Guerra and M. d. L. Centeno. Are quantile risk measures suitable for risk-transfer decisions? Insurance: Math. Econom., 50(3):446-461, 2012.

[22] M. Kaluszka. Optimal reinsurance under mean-variance premium principles. Insurance: Math. Econom., 28(1):61-67, 2001.

[23] A. Kull. Sharing risk - an economic perspective. Astin Bull., 39(2):591-613, 2009.

[24] A. Lo. A Neyman-Pearson perspective on optimal reinsurance with constraints. ASTIN Bull., 47(2):467-499, 2017.

[25] S. Malamud, H. Rui, and A. Whinston. Optimal reinsurance with multiple tranches. J. Math. Econom., 65:71-82, 2016.

[26] K. S. Tan, C. Weng, and Y. Zhang. VaR and CTE criteria for optimal quota-share and stop-loss reinsurance. North American Actuarial Journal, 13(4):459-482, 2009.

E-mail address: hansjoerg.albrecher@unil.ch

University of Lausanne and Swiss Finance Institute, Quartier UNIL-Dorigny, Bâtiment Extranef, 1015 Lausanne, Switzerland

E-mail address: arian.cani@unil.ch

University of Lausanne, Quartier UniL-Dorigny, BÂtiment Extranef, 1015 LaUSANNE, SWITZERLAND 